\title{
A big symmetric planar set with small category projections
}

\author{
by \\ Krzysztof Ciesielski (Morgantown, WV) and \\ Tomasz Natkaniec (Gdańsk)
}

\begin{abstract}
We show that under appropriate set-theoretic assumptions (which follow from Martin's axiom and the continuum hypothesis) there exists a nowhere meager set $A \subset \mathbb{R}$ such that

(i) the set $\{c \in \mathbb{R}: \pi[(f+c) \cap(A \times A)]$ is not meager $\}$ is meager for each continuous nowhere constant function $f: \mathbb{R} \rightarrow \mathbb{R}$,

(ii) the set $\{c \in \mathbb{R}:(f+c) \cap(A \times A)=\emptyset\}$ is nowhere meager for each continuous function $f: \mathbb{R} \rightarrow \mathbb{R}$.

The existence of such a set also follows from the principle CPA, which holds in the iterated perfect set model. We also prove that the existence of a set $A$ as in (i) cannot be proved in $\mathrm{ZFC}$ alone even when we restrict our attention to homeomorphisms of $\mathbb{R}$. On the other hand, for the class of real-analytic functions a Bernstein set $A$ satisfying (ii) exists in ZFC.
\end{abstract}

1. The results. Let $\mathcal{M}$ denote the class of all meager subsets of $\mathbb{R}$ and let $\pi: \mathbb{R}^{2} \rightarrow \mathbb{R}$ be the projection onto the first coordinate. For a function $f: \mathbb{R} \rightarrow \mathbb{R}$ and a set $E \subset \mathbb{R}^{2}$ the $f$-category projection of $E$ is the set $\{c \in \mathbb{R}: \pi[(f+c) \cap E] \notin \mathcal{M}\}$. (See [CG].) In papers [Da], [N1], and [N2] their authors considered the second category sets $A \subset \mathbb{R}$ such that an $f$ category projection of $A \times A$ has an empty interior for every linear function $f(x)=a x+b$. Inspired by these results Bartoszyński and Halbeisen [BH] recently constructed a second category set $A \subset \mathbb{R}$ with an even stronger property: for each polynomial $p$ which is neither constant nor the identity

2000 Mathematics Subject Classification: Primary 03E35; Secondary 26A99, 03E50.

Key words and phrases: $f$-category projections, transfinite induction, nowhere meager sets, Covering Property Axiom CPA, $\omega_{1}$-oracle.

The work of K. Ciesielski was partially supported by NATO Grant PST.CLG.977652 and 2002/03 West Virginia University Senate Research Grant.

The work of T. Natkaniec was partially supported by Gdańsk University Research Grant No. BW 5100-5-02331-2. 
function the set $p \cap(A \times A)$ is finite. In particular, if $p$ is a non-constant polynomial then the $p$-category projection of $A \times A$ has an empty interior.

These results lead to the following, more general question: for which classes $\mathcal{F}$ of continuous functions does there exist a "big" set $A \subset \mathbb{R}$ such that the $f$-category projection is "small" for every $f \in \mathcal{F}$ ? In particular, what happens for the classes $\mathcal{A}$ of real-analytic functions, $\mathcal{C}_{0}$ of all nowhere constant functions from $\mathbb{R}$ to $\mathbb{R}$, and for the entire class $\mathcal{C}$ of all continuous functions from $\mathbb{R}$ to $\mathbb{R}$ ?

In this note we answer these questions. We use standard terminology as in [Ci]. We consider only real-valued functions of one variable, unless otherwise specified. No distinction is made between a function and its graph. For functions $f, g$ put $[f=g]=\{x \in \mathbb{R}: f(x)=g(x)\}$. A set $A \subset \mathbb{R}$ is nowhere meager if $A \cap I \notin \mathcal{M}$ for each non-degenerate interval $I$. The symbol $B(x, r)$ denotes the open ball centered at $x$ and with radius $r$, and id stands for the identity function.

The main general theorem in the positive direction is the following result.

Theorem 1. Let $\mathcal{J} \supset[\mathbb{R}] \leq \omega$ be a translation invariant ideal on $\mathbb{R}$ and $\mathcal{B}_{0}$ be a family of Borel sets containing all Borel non-meager sets such that

$$
B \backslash \bigcup \mathcal{G} \neq \emptyset \quad \text { for every } B \in \mathcal{B}_{0} \text { and } \mathcal{G} \in[\mathcal{J}]^{<\mathfrak{c}} .
$$

If $\mathcal{F} \subset \mathcal{C}$ is such that for every $f \in \mathcal{F}$,

$$
\text { the set } L_{f}=\left\{y \in \mathbb{R}: f^{-1}(y) \notin \mathcal{J}\right\} \text { belongs to } \mathcal{J}
$$

then there exists an $A \subset \mathbb{R}$ intersecting every $B \in \mathcal{B}_{0}$ such that for every $f \in \mathcal{F}$,

(a) there exists a $Z \in[\mathbb{R}]^{<\mathfrak{c}}$ such that $f \cap(A \times A) \subset(A \times Z) \cup \mathrm{id}$. Moreover, if (2) holds for every $f \in( \pm \mathcal{F}) \cup(\mathrm{id}-\mathcal{F})$ then we can also have the following property for every $f \in \mathcal{F}$ :

(b) the set $\{c \in \mathbb{R}:(f+c) \cap(A \times A)=\emptyset\}$ intersects every $B \in \mathcal{B}_{0}$.

We leave the proof of this theorem to the next section. In the remainder of this section we discuss several of its corollaries. In particular, applying Theorem 1 to the ideal $[\mathbb{R}]^{\leq \omega}$ of countable sets and the family $\mathcal{B}_{0}$ of all uncountable Borel sets we get the following result.

Corollary 2. Let $\mathcal{F} \subset \mathcal{C}$ be such that the set $L_{f}=\left\{y \in \mathbb{R}:\left|f^{-1}(y)\right|>\omega\right\}$ is empty for every $f \in \mathcal{F}$. Then there exists a Bernstein set $A \subset \mathbb{R}$ such that for every $f \in \mathcal{F}$,

(a) there exists a $Z \in[\mathbb{R}]^{<\mathfrak{c}}$ such that $f \cap(A \times A) \subset(A \times Z) \cup$ id. In addition, if $L_{f}=\emptyset$ for every $f \in( \pm \mathcal{F}) \cup(\mathrm{id}-\mathcal{F})$ then we can also have

(b) $\{c \in \mathbb{R}:(f+c) \cap(A \times A)=\emptyset\}$ contains a Bernstein set for every $f \in \mathcal{F}$. 
In particular,

- there exists a set $A$ satisfying (a) for every countable-to-one function $f \in \mathcal{C}$;

- there exists a set $A$ satisfying (a) and (b) for every analytic function $f \in \mathcal{A}$; moreover, if $f \neq \mathrm{id}$ is not constant then $|f \cap(A \times A)|<\mathfrak{c}$.

Proof. Clearly the ideal $\mathcal{J}=[\mathbb{R}] \leq \omega$ and the family $\mathcal{B}_{0}$ of all uncountable Borel sets satisfy (1) from Theorem 1. Since we assume that (2) is also satisfied, the first assertion of the corollary follows from Theorem 1 . To see the "additional" part for analytic functions first notice that $\mathcal{F}=\mathcal{A}$ satisfies the assumption for (b), since $L_{f}=\emptyset$ for every non-constant $f \in \mathcal{A}$. In particular, if $f \neq \mathrm{id}$, then by (a) the set $\pi[f \cap(A \times A)]$ is contained in $(f-\mathrm{id})^{-1}(0) \cup \bigcup_{z \in Z} f^{-1}(z)$, which is a union of less than $\mathfrak{c}$ countable sets.

In the proof of the next corollary we need the following simple fact:

If $f \in \mathcal{C}$ then the set $\{c:[f+c=\mathrm{id}] \notin \mathcal{M}\}$ is countable.

To see it notice that for each $c \in \mathbb{R}$ the set $[f+c=\mathrm{id}]$ is closed. Therefore, if $[f+c=\mathrm{id}] \notin \mathcal{M}$, then there exists a non-empty open interval $I_{c}$ with $f(x)=x-c$ for each $x \in I_{c}$. It is easy to observe that $I_{c} \cap I_{d}=\emptyset$ if $c \neq d$.

COROllary 3. There exists a Bernstein set $A \subset \mathbb{R}$ such that for every homeomorphism $f: \mathbb{R} \rightarrow \mathbb{R}$ for all but countably many $c \in \mathbb{R}$ the set $\pi[(f+c) \cap(A \times A)]$ is a union of a meager set and a set of cardinality less than $\mathfrak{c}$. In particular, if $[\mathbb{R}]^{<\mathfrak{c}} \subset \mathcal{M}$ then the set

$$
\{c \in \mathbb{R}: \pi[(f+c) \cap(A \times A)] \notin \mathcal{M}\}
$$

is countable (so meager) for every homeomorphism $f: \mathbb{R} \rightarrow \mathbb{R}$.

Proof. Let $A$ be as in Corollary 2. So for every homeomorphism $f: \mathbb{R} \rightarrow \mathbb{R}$ and $c \in \mathbb{R}$ there exists a $Z \in[\mathbb{R}]^{<\mathfrak{c}}$ such that $(f+c) \cap(A \times A) \subset(A \times Z) \cup$ id. Notice that $\pi[(f+c) \cap(A \times A)] \subset[f+c=\mathrm{id}] \cup \pi[(f+c) \cap(A \times Z)]$ and $\pi[(f+c) \cap(A \times Z)] \subset f^{-1}(Z-c) \in[\mathbb{R}]^{<\mathfrak{c}}$. So there exists a $D \in[\mathbb{R}]^{<\mathfrak{c}}$ such that $\pi[(f+c) \cap(A \times A)] \subset[f+c=\mathrm{id}] \cup D$. The conclusion follows from (3).

Notice that the set-theoretic assumption in Corollary 3 is essential.

THEOREM 4. It is relatively consistent with ZFC that for every nowhere meager set $A \subset \mathbb{R}$ there exists a homeomorphism $f: \mathbb{R} \rightarrow \mathbb{R}$ such that the set $\{c \in \mathbb{R}: \pi[(f+c) \cap(A \times A)] \notin \mathcal{M}\}$ is nowhere meager.

Proof. This follows immediately from Theorem 12 and Proposition 13, which will be proven in Section 4.

Applying Theorem 1 to the ideal $\mathcal{M}$ and the family $\mathcal{B}_{0}$ of non-meager Borel sets we also get the following result. (Recall that the set-theoretic 
assumption about covering by meager sets follows form Martin's axiom MA and from the continuum hypothesis $\mathrm{CH}$.)

Corollary 5. Assume that less than continuum many meager sets do not cover $\mathbb{R}$. Then there exists a nowhere meager set $A \subset \mathbb{R}$ such that for every $f \in \mathcal{C}$ the set $\{c \in \mathbb{R}:(f+c) \cap(A \times A)=\emptyset\}$ is nowhere meager in $\mathbb{R}$. In particular, the $f$-category projection of $A \times A$ has an empty interior.

Proof. The set-theoretic assumption ensures that the ideal $\mathcal{J}=\mathcal{M}$ and the family $\mathcal{B}_{0}$ of non-meager Borel sets satisfy assumption (1) of Theorem 1. Since for $\mathcal{J}=\mathcal{M},(2)$ holds for every $f \in \mathcal{C}$, there is a set $A$ satisfying (b) from Theorem 1. Clearly, it has the desired properties.

For the class $\mathcal{C}_{0}$ of nowhere constant functions we have yet another corollary. In its proof we will use the following simple fact:

$$
f^{-1}(M) \in \mathcal{M} \quad \text { for every } f \in \mathcal{C}_{0} \text { and } M \in \mathcal{M} .
$$

Indeed, if sets $F_{n}$ are closed and nowhere dense in $\mathbb{R}$ such that $M \subset \bigcup_{n<\omega} F_{n}$ then $f^{-1}(M) \subset \bigcup_{n<\omega} f^{-1}\left(F_{n}\right)$. It is enough to notice that $f^{-1}\left(F_{n}\right)$ is closed and nowhere dense for every $f \in \mathcal{C}_{0}$.

Corollary 6. Assume that less than continuum many meager sets do not cover $\mathbb{R}$ and that $[\mathbb{R}]^{<\mathfrak{c}} \subset \mathcal{M}$. Then there exists a nowhere meager set $A \subset \mathbb{R}$ such that for every $f \in \mathcal{C}_{0}$ the set $\{c \in \mathbb{R}: \pi[(f+c) \cap(A \times A)] \notin \mathcal{M}\}$ is countable.

Proof. Let $f \in \mathcal{C}_{0}$ and $c \in \mathbb{R}$. By Theorem 1 (a) there exists a $Z \in[\mathbb{R}]^{<\mathfrak{c}}$ such that $\pi[(f+c) \cap(A \times A)] \subset[f+c=\mathrm{id}] \cup \pi[(f+c) \cap(A \times Z)]$. But, by (4), $\pi[(f+c) \cap(A \times Z)] \subset f^{-1}(Z-c) \in \mathcal{M}$ since $Z-c \in[\mathbb{R}]^{<\mathfrak{c}} \subset \mathcal{M}$. Thus, $\pi[(f+c) \cap(A \times A)] \in \mathcal{M}$ as long as $[f+c=\mathrm{id}] \in \mathcal{M}$. So the result follows immediately from $(3)$.

We believe that the conclusion of Corollary 6 cannot be proved in ZFC; we state this below as a conjecture. (See also the last section of the paper for some comments on it.)

ConjeCture 1. It is relatively consistent with ZFC that for every nowhere meager set $A \subset \mathbb{R}$ there is an $f \in \mathcal{C}_{0}$ such that $\pi[(f+c) \cap(A \times A)] \notin \mathcal{M}$ for every $c \in \mathbb{R}$.

It is worth noting that a set $A$ as in Corollaries 5 and 6 can also be constructed under the Covering Property Axiom CPA, which extracts the essence of the iterated perfect set model. (See [CP2, CMP, CP3].) This is of interest, since under CPA the set-theoretic assumptions of each of these corollaries are false: CPA implies that $\mathfrak{c}=\omega_{2}$ and that $\mathbb{R}$ can be covered by $\omega_{1}$ meager sets. In fact, in the theorem we will use only a simpler version of CPA known as $\mathrm{CPA}_{\text {cube }}^{\text {game }}$. 


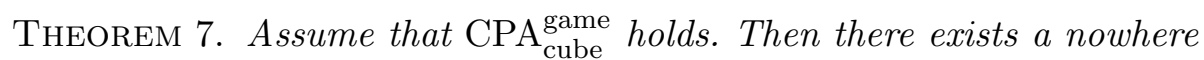
meager set $A \subset \mathbb{R}$ of cardinality $\omega_{1}<\mathfrak{c}$ such that for every $f \in \mathcal{C}$ there exists a countable set $Z \subset A$ such that

$$
f \cap(A \times A) \subset(A \times Z) \cup \mathrm{id} .
$$

In particular, for every $f \in \mathcal{C}_{0}$ the set $\{c \in \mathbb{R}: \pi[(f+c) \cap(A \times A)] \notin \mathcal{M}\}$ is countable and the set $\{c \in \mathbb{R}:(f+c) \cap(A \times A)=\emptyset\}$ is the complement of a set of cardinality $\omega_{1}<\mathfrak{c}$, so it contains a Bernstein set.

Proof. The first assertion will be proved in Section 3. To prove the second assertion, fix an $f \in \mathcal{C}_{0}$. The proof that $\{c \in \mathbb{R}: \pi[(f+c) \cap(A \times A)] \notin \mathcal{M}\}$ is countable is exactly the same as for Corollary 6 . To see that the complement $D$ of $\{c \in \mathbb{R}:(f+c) \cap(A \times A)=\emptyset\}$ has cardinality $\omega_{1}$ notice that if $(f+c) \cap(A \times A) \neq \emptyset$ then there are $x, y \in A$ such that $f(x)+c=y$, that is, $c=y-f(x) \in A-f[A]$. So $D$ equals $A-f[A]$ and has cardinality $\omega_{1}$.

It is also important to notice that the set $A$ in Corollaries 5 and 6 cannot have the Baire property.

Proposition 8. Suppose that $f \in \mathcal{C}_{0}$ and that $A \subset \mathbb{R}$ is a non-meager set having the Baire property. Then the $f$-category projection of $A \times A$ has a non-void interior.

Proof. Let $G$ be a non-empty open set with $M=G \backslash A \in \mathcal{M}$. Fix $x_{0} \in G \cap A$ and $c_{0} \in \mathbb{R}$ with $f\left(x_{0}\right)+c_{0} \in G \cap A$, an $\varepsilon>0$ such that $B\left(f\left(x_{0}\right)+c_{0}, 2 \varepsilon\right) \subset G$, and a $\delta>0$ such that $f\left[B\left(x_{0}, \delta\right)\right] \subset B\left(f\left(x_{0}\right), \varepsilon\right)$. Let $c \in B\left(c_{0}, \varepsilon\right)$. Since $A_{0}=A \cap B\left(x_{0}, \delta\right) \notin \mathcal{M}$ and $f+c \in \mathcal{C}_{0}$, condition (4) implies $(f+c)\left(A_{0}\right) \notin \mathcal{M}$. Since $(f+c)\left(A_{0}\right) \subset G$ and $(f+c)\left(A_{0}\right) \cap A \notin \mathcal{M}$, it follows that $c$ belongs to the $f$-category projection of $A \times A$.

We finish this section with the following result of Bartoszyński and Halbeissen, which was one of the starting points for this note.

THEOREM $9([\mathrm{BH}])$. There exists a set $A \subset \mathbb{R}$ intersecting every perfect set such that for each non-constant polynomial $p \neq \mathrm{id}$ the set $p \cap(A \times A)$ is finite.

Note that Corollary 2 implies immediately a weaker version of Theorem 9: there exists a Bernstein set $A$ for which each set $p \cap(A \times A)$ has cardinality less than $\mathfrak{c}$. However, we see no easy way to deduce the full version of the theorem from the results presented above. Nevertheless we wish to include here a very short proof of Theorem 9, since it is considerably simpler and completely different from the argument presented in $[\mathrm{BH}]$.

Proof. First notice that if $A$ is a transcendental base of $\mathbb{R}$ (over $\mathbb{Q}$ ) then the set $p \cap(A \times A)$ is finite for every polynomial $p$ which is neither constant nor the identity function. Indeed, if $K \in[A]^{<\omega}$ is such that $p \in \overline{\mathbb{Q}(K)}[x]$, where $\overline{\mathbb{Q}(K)}$ stands for the algebraic closure of $\mathbb{Q}(K)$ in $\mathbb{R}$, then for every 
$a \in A \backslash K$ we have $p(a) \in \overline{\mathbb{Q}(K \cup\{a\})} \backslash \overline{\mathbb{Q}(K)}$, since $A$ is algebraically independent. (See e.g. [Ku, Lemma 2, p. 99].) So if $p(a) \in A$ then $p(a)=a$. But this is impossible, since $p(a)=a$ implies that $a$ is a root of a non-zero polynomial $p-$ id $\in \overline{\mathbb{Q}(K)}[x]$. So $\pi[p \cap(A \times A)] \subset K$. It is well known that there are transcendental bases $A$ that are also Bernstein sets $([\mathrm{Ci}$, Corollary 7.3.6 and Exercise 2 on page 126]) and any such base satisfies the conclusion.

2. Proof of Theorem 1. Let $\left\{\left\langle f_{\alpha}, B_{\alpha}\right\rangle: \alpha<\mathfrak{c}\right\}$ be an enumeration of $\mathcal{F} \times \mathcal{B}_{0}$. For each $\alpha<\mathfrak{c}$ we will choose, by induction on $\alpha<\mathfrak{c}$, points $x_{\alpha} \in B_{\alpha}$ and $c_{\alpha} \in B_{\alpha}$ aiming for $A=\left\{x_{\alpha}: \alpha<\mathfrak{c}\right\}$. We will set up the induction in such a way that for every $\alpha<\mathfrak{c}$ the set $Z$ satisfying (a) for $f_{\alpha}$ will be $A_{\alpha}=\left\{x_{\beta}: \beta<\alpha\right\}$ and, if $(2)$ holds for every $f \in( \pm \mathcal{F}) \cup($ id $-\mathcal{F})$, that $\left(f_{\alpha}+c_{\alpha}\right) \cap(A \times A)=\emptyset$. So assume that for some $\alpha<\mathfrak{c}$ the sets $\left\{x_{\beta}: \beta<\alpha\right\}$ and $\left\{c_{\beta}: \beta<\alpha\right\}$ are already constructed. If we need only ensure (a), we put $c_{\alpha}=x_{\alpha}$ and choose an $x_{\alpha} \in B_{\alpha} \backslash \bigcup_{\beta \leq \alpha} L_{f_{\beta}}$ such that

$$
f_{\beta} \cap\left(\left\{x_{\alpha}\right\} \times\left(A_{\alpha} \backslash A_{\beta}\right)\right)=\emptyset=f_{\beta} \cap\left(A_{\alpha} \times\left\{x_{\alpha}\right\}\right)
$$

for all $\beta \leq \alpha$. This is possible by (1), since $\left\{L_{f_{\beta}}: \beta \leq \alpha\right\} \subset \mathcal{J}$, the singletons $f_{\beta}[\{a\}]$ are in $\mathcal{J}$ for $\beta \leq \alpha$ and $a \in A_{\alpha}$, and $\left\{x \in \mathbb{R}: f_{\beta}(x)=x_{\gamma}\right\}=$ $f_{\beta}^{-1}\left(x_{\gamma}\right) \in \mathcal{J}$ for every $\beta \leq \gamma<\alpha$ since $x_{\gamma} \notin L_{f_{\beta}}$. It is easy to see that such a choice implies that $f_{\alpha} \cap(A \times A) \subset\left(A \times A_{\alpha}\right) \cup$ id for every $\alpha<\mathfrak{c}$. So in what follows we assume that $(2)$ holds for every $f \in( \pm \mathcal{F}) \cup(\mathrm{id}-\mathcal{F})$. We also assume that the following inductive conditions hold for every $\alpha<\mathfrak{c}$ :

(I) $\left(\text { id }-f_{\alpha}\right)^{-1}\left(c_{\alpha}\right) \cup f_{\alpha}^{-1}\left(x_{\beta}-c_{\alpha}\right) \in \mathcal{J}$ for every $\beta<\alpha$,

(II) $f_{\beta}^{-1}\left(x_{\alpha}\right) \cup f_{\beta}^{-1}\left(x_{\alpha}-c_{\beta}\right) \in \mathcal{J}$ for every $\beta \leq \alpha$.

First choose a $c_{\alpha} \in B_{\alpha} \backslash\left(L_{\mathrm{id}-f_{\alpha}} \cup \bigcup_{\beta<\alpha}\left(x_{\beta}+L_{-f_{\alpha}}\right)\right)$ outside the set

$$
\left\{c \in \mathbb{R}:\left(f_{\alpha}+c\right) \cap\left(A_{\alpha} \times A_{\alpha}\right) \neq \emptyset\right\}=A_{\alpha}-f_{\alpha}\left[A_{\alpha}\right] \in[\mathbb{R}]^{<\mathfrak{c}} .
$$

Such a choice is possible by (1), since the singletons and the sets $L_{\mathrm{id}-f_{\alpha}}$ and $x_{\beta}+L_{-f_{\alpha}}$ belong to $\mathcal{J}$. Clearly $c_{\alpha} \notin L_{\mathrm{id}-f_{\alpha}}$ ensures that $\left(\mathrm{id}-f_{\alpha}\right)^{-1}\left(c_{\alpha}\right) \in \mathcal{J}$. Similarly, $c_{\alpha} \notin x_{\beta}+L_{-f_{\alpha}}$ implies that $c_{\alpha}-x_{\beta} \notin L_{-f_{\alpha}}$ and hence the set $f_{\alpha}^{-1}\left(x_{\beta}-c_{\alpha}\right)=\left(-f_{\alpha}\right)^{-1}\left(c_{\alpha}-x_{\beta}\right)$ belongs to $\mathcal{J}$. Thus, condition (I) is satisfied. Note also that $c_{\alpha} \notin A_{\alpha}-f_{\alpha}\left[A_{\alpha}\right]$ implies that

(i) $\left(f_{\alpha}+c_{\alpha}\right) \cap\left(A_{\alpha} \times A_{\alpha}\right)=\emptyset$.

Next choose an $x_{\alpha} \in B_{\alpha} \backslash \bigcup_{\beta \leq \alpha}\left(L_{f_{\beta}} \cup\left(c_{\beta}+L_{f_{\beta}}\right)\right)$ such that

$$
\left(f_{\beta}+c_{\beta}\right) \cap\left(\left\{x_{\alpha}\right\} \times A_{\alpha}\right)=\emptyset=\left(f_{\beta}+c_{\beta}\right) \cap\left(A_{\alpha} \times\left\{x_{\alpha}\right\}\right),
$$

$x_{\alpha} \notin \bigcup_{\gamma \leq \alpha}\left(\mathrm{id}-f_{\gamma}\right)^{-1}\left(c_{\gamma}\right)$, and

$$
f_{\beta} \cap\left(\left\{x_{\alpha}\right\} \times\left(A_{\alpha} \backslash A_{\beta}\right)\right)=\emptyset=f_{\beta} \cap\left(A_{\alpha} \times\left\{x_{\alpha}\right\}\right)
$$


for every $\beta \leq \alpha$. This is possible by (1), since $\left\{L_{f_{\beta}} \cup\left(c_{\beta}+L_{f_{\beta}}\right): \beta \leq \alpha\right\} \subset \mathcal{J}$ and by inductive assumptions (I)\&(II) for every $a \in A_{\alpha}$ and $\beta \leq \gamma<\alpha$ the following sets belong to $\mathcal{J}$ :

- $\left\{x \in \mathbb{R}:\left(f_{\beta}+c_{\beta}\right)(x)=a\right\}=f_{\beta}^{-1}\left(a-c_{\beta}\right)$;

- $\left(f_{\beta}+c_{\beta}\right)[\{a\}]$;

- $\left(\mathrm{id}-f_{\gamma}\right)^{-1}\left(c_{\gamma}\right)$;

- $\left\{x \in \mathbb{R}: f_{\beta}(x)=x_{\gamma}\right\}=f_{\beta}^{-1}\left(x_{\gamma}\right)$;

- $f_{\beta}[\{a\}]$.

Note that $x_{\alpha} \notin \bigcup_{\beta \leq \alpha} L_{f_{\beta}}$ ensures $f_{\beta}^{-1}\left(x_{\alpha}\right) \in \mathcal{J}$, and $x_{\alpha} \notin \bigcup_{\beta \leq \alpha}\left(c_{\beta}+L_{f_{\beta}}\right)$ implies that $x_{\alpha}-c_{\beta} \notin L_{f_{\beta}}$, so the set $f_{\beta}^{-1}\left(x_{\alpha}-c_{\beta}\right)$ is in $\mathcal{J}$. In particular, (II) is satisfied. This finishes the inductive construction.

Clearly $A$ is nowhere meager, since it meets every non-meager Borel set. To see that (b) holds notice that by (i) we have $\left(f_{\beta}+c_{\beta}\right) \cap\left(A_{\beta} \times A_{\beta}\right)=\emptyset$ while $\left(f_{\beta}+c_{\beta}\right) \cap\left(A_{\alpha} \times A_{\alpha}\right)=\emptyset$ for $\alpha>\beta$ is ensured by the choice of $x_{\alpha}$ as in (5) and the fact that $\left(f_{\beta}+c_{\beta}\right)\left(x_{\alpha}\right) \neq x_{\alpha}$ since $x_{\alpha} \notin\left(\mathrm{id}-f_{\beta}\right)^{-1}\left(c_{\beta}\right)$. To see that (a) holds pick an $f \in \mathcal{F}$ and let $\beta<\mathfrak{c}$ be such that $f=f_{\beta}$. The choice of $x_{\alpha}$ for $\alpha>\beta$ as in (6) implies that $f \cap(A \times A) \subset\left(A \times A_{\beta}\right) \cup$ id.

3. Set $A$ from CPA. To formulate axiom $\mathrm{CPA}_{\text {cube }}^{\text {game }}$ we need a few definitions. Let $\mathfrak{C}$ denote the Cantor set $2^{\omega}$. For a Polish space $X$ we use $\operatorname{Perf}(X)$ to denote the family of all subsets of $X$ homeomorphic to $\mathfrak{C}$. A subset $C$ of a product $\mathfrak{C}^{\omega}$ of the Cantor set is said to be a perfect cube if $C=\prod_{n \in \omega} C_{n}$, where $C_{n} \in \operatorname{Perf}(\mathfrak{C})$ for each $n$. For a fixed Polish space $X$ let $\mathcal{F}_{\text {cube }}$ stand for the family of all continuous injections from a perfect cube $C \subset \mathfrak{C}^{\omega}$ onto a set $P$ from $\operatorname{Perf}(X)$. We consider each function $f \in \mathcal{F}_{\text {cube }}$ from $C$ onto $P$ as a coordinate system imposed on $P$.

We say that $P \in \operatorname{Perf}(X)$ is a cube if we consider it with (implicitly given) witness function $f \in \mathcal{F}_{\text {cube }}$ onto $P$, and $Q$ is a subcube of a cube $P \in \operatorname{Perf}(X)$ provided $Q=f[C]$, where $f \in \mathcal{F}_{\text {cube }}$ is a witness function for $P$ and $C \subset \operatorname{dom}(f) \subset \mathfrak{C}^{\omega}$ is a perfect cube. (Here and in what follows $\operatorname{dom}(f)$ stands for the domain of $f$.)

We say that a family $\mathcal{E} \subset \operatorname{Perf}(X)$ is cube dense in $\operatorname{Perf}(X)$ if every cube $P \in \operatorname{Perf}(X)$ contains a subcube $Q \in \mathcal{E}$. More formally, $\mathcal{E} \subset \operatorname{Perf}(X)$ is cube dense provided

$$
\forall f \in \mathcal{F}_{\text {cube }} \exists g \in \mathcal{F}_{\text {cube }}(g \subset f \& \operatorname{range}(g) \in \mathcal{E}) .
$$

We also need a notion of a constant cube: the family $\mathcal{C}_{\text {cube }}(X)$ of constant "cubes" is defined as the family of all constant functions from a perfect cube $C \subset \mathfrak{C}^{\omega}$ to $X$. We define $\mathcal{F}_{\text {cube }}^{*}(X)$ as

$$
\mathcal{F}_{\text {cube }}^{*}=\mathcal{F}_{\text {cube }} \cup \mathcal{C}_{\text {cube }} \text {. }
$$


Thus, $\mathcal{F}_{\text {cube }}^{*}$ is the family of all continuous functions from a perfect cube $C \subset \mathfrak{C}^{\omega}$ into $X$ which are either one-to-one or constant. Now the range of every $f \in \mathcal{F}_{\text {cube }}^{*}$ belongs to the family $\operatorname{Perf}^{*}(X)$ of all sets $P$ such that either $P \in \operatorname{Perf}(X)$ or $P$ is a singleton. The meaning of " $P \in \operatorname{Perf}^{*}(X)$ is a cube" and " $Q$ is a subcube of a cube $P \in \operatorname{Perf}^{*}(X)$ " is defined in a natural way.

For a Polish space $X$ consider the following game $\operatorname{GAME}_{\text {cube }}(X)$ of length $\omega_{1}$. The game has two players, I and II. At each stage $\xi<\omega_{1}$ of the game Player I can play an arbitrary cube $P_{\xi} \in \operatorname{Perf}^{*}(X)$ and Player II must respond with a subcube $Q_{\xi}$ of $P_{\xi}$. The game $\left\langle\left\langle P_{\xi}, Q_{\xi}\right\rangle: \xi<\omega_{1}\right\rangle$ is won by Player I provided

$$
\bigcup_{\xi<\omega_{1}} Q_{\xi}=X
$$

otherwise the game is won by Player II. A strategy for Player II is any function $S$ such that $S\left(\left\langle\left\langle P_{\eta}, Q_{\eta}\right\rangle: \eta<\xi\right\rangle, P_{\xi}\right)$ is a subcube of $P_{\xi}$, where $\left\langle\left\langle P_{\eta}, Q_{\eta}\right\rangle: \eta<\xi\right\rangle$ is any partial game. (We abuse here slightly the notation, since the function $S$ also depends on the implicitly given coordinate functions $f_{\eta}: \mathfrak{C}^{\omega} \rightarrow P_{\eta}$ making each $P_{\eta}$ a cube.) A game $\left\langle\left\langle P_{\xi}, Q_{\xi}\right\rangle: \xi<\omega_{1}\right\rangle$ is played according to a strategy $S$ provided $Q_{\xi}=S\left(\left\langle\left\langle P_{\eta}, Q_{\eta}\right\rangle: \eta<\xi\right\rangle, P_{\xi}\right)$ for every $\xi<\omega_{1}$. A strategy $S$ for Player II is a winning strategy for Player II provided Player II wins any game played according to $S$. Now we can formulate the following axiom (see [CP3]):

$\mathrm{CPA}_{\text {cube }}^{\text {game }} \mathfrak{c}=\omega_{2}$ and for any Polish space $X$ Player II has no winning strategy in the game $\mathrm{GAME}_{\text {cube }}(X)$.

All we need to know about cube-dense families is the following fact.

FACT 10. Let $X$ be a Polish space and let $\mathcal{E} \subset \operatorname{Perf}^{*}(X)$ contain all singletons. If for every $P \in \operatorname{Perf}(X)$ and every Borel probability measure $\mu$ on $P$ there exists a $Q \in \operatorname{Perf}(P) \cap \mathcal{E}$ such that $\mu(Q)>0$, then $\mathcal{E}$ is cube-dense.

Proof. This follows immediately from [CP1, Claim 3.2]. (See also [CP3, Claim 1.1.5] or [CP2, Claim 2.3].)

We will apply this fact to $X=\mathcal{C}$, where $\mathcal{C}$ is considered with the sup norm. Notice that for every $Q \subset \mathcal{C}$ the set $\bigcup Q \subset \mathbb{R}^{2}$ is the union of the graphs of all functions belonging to $Q$, since functions are identified with their graphs. In what follows, for a set $K \subset \mathbb{R}^{2}$ and $x \in \mathbb{R}$ we denote by $K_{x}$ the vertical section of $K$ above $x$, that is, $K_{x}=\{y:\langle x, y\rangle \in K\}$. Similarly, $K^{x}=\{x:\langle x, y\rangle \in K\}$. For $A \in[\mathbb{R}] \leq \omega$ let $\mathcal{E}(A)$ be the family of all $Q \in \operatorname{Perf}^{*}(\mathcal{C})$ such that

- $\bigcup Q$ is nowhere dense in $\mathbb{R}^{2}$,

- $[\bigcup Q]_{x}$ is nowhere dense in $\mathbb{R}$ for every $x \in A$. 
Lemma 1. The family $\mathcal{E}(A)$ is cube-dense for every $A \in[\mathbb{R}] \leq \omega$.

Proof. Without loss of generality we can assume that $A$ is dense in $\mathbb{R}$. Clearly every singleton belongs to $\mathcal{E}(A)$. So let $P \in \operatorname{Perf}(\mathcal{C})$ and let $\mu$ be a Borel probability measure $\mu$ on $P$. By Fact 10 it is enough to show that there exists a $Q \in \operatorname{Perf}(P) \cap \mathcal{E}(A)$ such that $\mu(Q)>0$. To see this, fix a countable base $\mathcal{B}$ for $\mathbb{R}$ and let $\left\langle\left\langle a_{n}, J_{n}\right\rangle: n<\omega\right\rangle$ be an enumeration of $A \times \mathcal{B}$. Notice that for every $n<\omega$ there exists a non-empty open set $U_{n} \subset J_{n}$ such that

$$
\mu\left(\left\{f \in P: f\left(a_{n}\right) \in U_{n}\right\}\right)<2^{-(n+2)} .
$$

Indeed, if $\mathcal{U}_{n}$ is an infinite family of non-empty pairwise disjoint open subsets of $J_{n}$ then for each $U \in \mathcal{U}_{n}$ the set $\left\{f \in P: f\left(a_{n}\right) \in U\right\}$ is open in $P$ (so $\mu$-measurable) and so condition (9) must hold for some $U \in \mathcal{U}_{n}$. Let $W=\bigcup_{n<\omega}\left\{f \in \mathcal{C}: f\left(a_{n}\right) \in U_{n}\right\}$. It is clear that $W$ is open and dense in $\mathcal{C}$. So $Q=P \backslash W=P \backslash \bigcup_{n<\omega}\left\{f \in P: f\left(a_{n}\right) \in U_{n}\right\}$ is nowhere dense (and therefore $\bigcup Q$ is nowhere dense in $\mathbb{R}^{2}$ ), and by (9), it has $\mu$-measure at least $1-\sum_{n<\omega} 2^{-(n+2)}=2^{-1}>0$. It is also clear that for every $x \in A$ the set $\bigcup\left\{U_{n}: a_{n}=x\right\}$ is dense open in $\mathbb{R}$ and it is disjoint from $[\bigcup Q]_{x}$. Thus $Q \in \mathcal{E}(A)$.

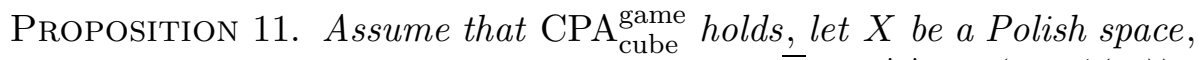
and let $S$ be a mapping associating to every $\bar{P} \in \bigcup_{\alpha<\omega_{1}}\left(\operatorname{Perf}^{*}(X)\right)^{\alpha}$ a cube-dense family $\mathcal{E}(\bar{P}) \subset \operatorname{Perf}^{*}(X)$. Then there exists a sequence $\left\langle\left\langle P_{\xi}, Q_{\xi}\right\rangle: \xi<\omega_{1}\right\rangle$ such that $Q_{\xi} \in \operatorname{Perf}^{*}\left(P_{\xi}\right) \cap \mathcal{E}\left(\left\langle P_{\zeta}: \zeta<\xi\right\rangle\right)$ for every $\xi<\omega_{1}$ and $X=\bigcup_{\xi<\omega_{1}} Q_{\xi}$.

Proof. This follows easily from $\mathrm{CPA}_{\text {cube }}^{\text {game }}$. More precisely, it is enough to apply $\mathrm{CPA}_{\text {cube }}^{\text {game }}$ to the strategy $S^{*}$ such that $S^{*}\left(\left\langle\left\langle P_{\eta}, Q_{\eta}\right\rangle: \eta<\xi\right\rangle, P_{\xi}\right)$ is a subcube of $P_{\xi}$ from $S\left(\left\langle P_{\eta}: \eta<\xi\right\rangle\right)$.

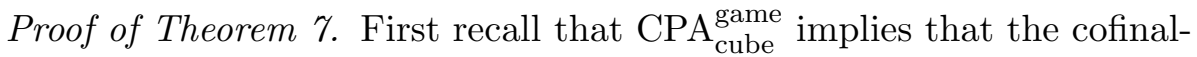
ity of the ideal of meager sets is equal to $\omega_{1}<\mathfrak{c}$, that is, there exists an $\mathcal{M}_{0} \in[\mathcal{M}]^{\omega_{1}}$ such that every meager set is contained in some $M \in \mathcal{M}_{0}$. (See e.g. [CP1, Sec. 4] or [CP3].) Let $\mathcal{B}_{0}$ be a countable base for $\mathbb{R}$ and let $\left\{\left\langle M_{\xi}, J_{\xi}\right\rangle: \xi<\omega_{1}\right\}$ be an enumeration of $\mathcal{M}_{0} \times \mathcal{B}_{0}$. By simultaneous induction on $\xi<\omega_{1}$, using Lemma 1, we will define functions $S, Q$, and $k$ on $\left(\operatorname{Perf}^{*}(\mathcal{C})\right)^{\xi}$ such that

(i) $S\left(\left\langle P_{\zeta}: \zeta<\xi\right\rangle\right)=\mathcal{E}\left(\left\{a_{\zeta}: \zeta<\xi\right\}\right)$, where $a_{\zeta}=k\left(\left\langle P_{\eta}: \eta \leq \zeta\right\rangle\right) \in \mathbb{R}$, and $Q_{\xi}=Q\left(\left\langle P_{\zeta}: \zeta<\xi\right\rangle\right) \in \mathcal{E}\left(\left\{a_{\zeta}: \zeta<\xi\right\}\right)$,

(ii) $k\left(\left\langle P_{\zeta}: \zeta \leq \xi\right\rangle\right)$ belongs to $J_{\xi}$ and to the residual set

$$
\bigcap_{\zeta \leq \xi}\left\{z \in \mathbb{R}:\left(\bigcup Q_{\zeta}\right)^{z} \text { and }\left(\bigcup Q_{\zeta}\right)_{z} \text { are nowhere dense in } \mathbb{R}\right\} \text {, }
$$


(iii) $k\left(\left\langle P_{\zeta}: \zeta \leq \xi\right\rangle\right)$ does not belong to the meager set

$$
M_{\xi} \cup \bigcup_{\eta \leq \xi}\left(\left\{\left(\bigcup Q_{\eta}\right)_{a_{\zeta}}: \zeta<\xi\right\} \cup\left\{\left(\bigcup Q_{\eta}\right)^{a_{\zeta}}: \eta \leq \zeta<\xi\right\}\right) .
$$

The set as in (ii) is residual by the Kuratowski-Ulam theorem, since each set $\bigcup Q_{\zeta}$ is nowhere dense, as $Q_{\zeta}$ belongs to some $\mathcal{E}(A)$. In (iii) for every $\eta \leq \zeta<\xi$ the set $\left(\bigcup Q_{\eta}\right)_{a_{\zeta}} \cup\left(\bigcup Q_{\eta}\right)^{a_{\zeta}}$ is nowhere dense by the choice of $a_{\zeta}=k\left(\left\langle P_{\eta}: \eta \leq \zeta\right\rangle\right)$ as in (ii). Finally, for $\zeta<\eta$ the set $\left(\bigcup Q_{\eta}\right)_{a_{\zeta}}$ is nowhere dense since, by (i), $Q_{\eta}$ belongs to $S\left(\left\langle P_{\zeta}: \zeta<\eta\right\rangle\right)=\mathcal{E}\left(\left\{a_{\zeta}: \zeta<\eta\right\}\right)$.

Now, by axiom $\mathrm{CPA}_{\text {cube }}^{\text {game }}$ and Proposition 11, there exists a sequence $\left\langle\left\langle P_{\xi}, Q_{\xi}, a_{\xi}\right\rangle: \xi<\omega_{1}\right\rangle$ such that $\mathcal{C}=\bigcup_{\xi<\omega_{1}} Q_{\xi}$ and conditions (i)-(iii) are satisfied. We claim that $A=\left\{a_{\xi}: \xi<\omega_{1}\right\}$ satisfies the conclusion of Theorem 7 .

Clearly, $A$ is nowhere meager since for every non-empty open set $U \subset \mathbb{R}$ and every meager set $M$ there exists a $\xi<\omega_{1}$ such that $J_{\xi} \subset U$ and $M \subset M_{\xi}$. But then $a_{\xi} \in\left(A \cap J_{\xi}\right) \backslash M_{\xi} \subset(A \cap U) \backslash M$, so $A \cap U \neq M$. To see the first assertion of Theorem 7 take an $f \in \mathcal{C}$. Then there exists an $\eta<\omega_{1}$ such that $f \in Q_{\eta}$. We claim that for $Z=\left\{a_{\beta}: \beta<\eta\right\}$ we have $f \cap(A \times A) \subset(A \times Z) \cup$ id. Indeed, let $\eta \leq \xi<\omega_{1}$ and $\zeta<\omega_{1}$ be such that $\zeta \neq \xi$. We need to show that $\left\langle a_{\zeta}, a_{\xi}\right\rangle \notin f$. But if $\zeta<\xi$ then, by (iii), $a_{\xi}$ does not belong to $\left[\bigcup Q_{\eta}\right]_{a_{\zeta}} \ni f\left(a_{\zeta}\right)$, so $\left\langle a_{\zeta}, a_{\xi}\right\rangle \notin f$. Similarly, if $\xi<\zeta$ then, again by (iii), $a_{\zeta}$ does not belong to $\left[\cup Q_{\eta}\right]^{a_{\xi}} \supset f^{-1}\left(a_{\xi}\right)$ and once more $\left\langle a_{\zeta}, a_{\xi}\right\rangle \notin f$.

4. Main consistency result. The main goal of this section is to prove the following theorem.

THEOREM 12. It is relatively consistent with $Z F C$ that $\mathfrak{c}=\omega_{2}$ and the following two conditions hold simultaneously:

(A) For every family $\left\{B_{\xi}: \xi<\omega_{1}\right\}$ of pairwise disjoint nowhere meager subsets of $\mathbb{R}^{2}$ there exists an increasing homeomorphism $f: \mathbb{R} \rightarrow \mathbb{R}$ such that $\pi\left[f \cap B_{\xi}\right]$ is nowhere meager for every $\xi<\omega_{1}$.

(B) Every nowhere meager set $B \subset \mathbb{R}$ contains a nowhere meager subset of cardinality $\omega_{1}$.

We use Theorem 12, in conjunction with the following proposition, to deduce Theorem 4 .

Proposition 13. Assume that $\mathfrak{c}>\omega_{1}$ and that (A) and (B) hold. Then for every nowhere meager set $A \subset \mathbb{R}$ there is a homeomorphism $f: \mathbb{R} \rightarrow \mathbb{R}$ such that $\{c \in \mathbb{R}: \pi[(f+c) \cap(A \times A)] \notin \mathcal{M}\}$ is nowhere meager.

Proof. By (B) we can assume that $|A|=\omega_{1}<\mathfrak{c}$. Let $B$ be a Bernstein set such that $(b+A) \cap\left(b^{\prime}+A\right)=\emptyset$ for any distinct $b, b^{\prime} \in B$. Let $\left\{c_{\xi}: \xi<\omega_{1}\right\}$ 
be a nowhere meager subset of $B$, let $B_{\xi}=A \times\left(c_{\xi}+A\right)$ for $\xi<\omega_{1}$, and let $f: \mathbb{R} \rightarrow \mathbb{R}$ be as in (A). Then $\{c \in \mathbb{R}: \pi[(f+c) \cap(A \times A)] \notin \mathcal{M}\}$ contains a nowhere meager set $\left\{-c_{\xi}: \xi<\omega_{1}\right\}$ since for every $\xi<\omega_{1}$ we have

$$
\pi\left[\left(f-c_{\xi}\right) \cap(A \times A)\right]=\pi\left[f \cap B_{\xi}\right] \notin \mathcal{M},
$$

finishing the proof.

The proof of Theorem 12 is a slight modification of the proof of the main result (Theorem 2) from [CS]. Also, Theorem 12 easily implies [CS, Theorem 2]. We will use the terminology and notation of [CS]. In particular, according to the machinery used in that paper, Theorem 12 follows in a standard way from the following lemma. (More precisely, condition (A) is ensured by the lemma, while (B) and $\mathfrak{c}=\omega_{2}$ are guaranteed by the iteration procedure.)

Lemma 2. For every family $\mathcal{B}=\left\{B_{\xi}: \xi<\omega_{1}\right\}$ of pairwise disjoint nowhere meager subsets of $\mathbb{R}^{2}$ and for every $\omega_{1}$-oracle $\mathcal{M}$ there exists an $\mathcal{M}$-cc forcing notion $Q_{\mathcal{B}}$ of cardinality $\omega_{1}$ such that $Q_{\mathcal{B}}$ forces

there exists an increasing homeomorphism $f: \mathbb{R} \rightarrow \mathbb{R}$ such that $\pi\left[f \cap B_{\xi}\right]$ is nowhere meager for every $\xi<\omega_{1}$.

In what follows we present the proof of Lemma 2. Let

$$
\Gamma=\left\{\lambda<\omega_{1}: \lambda \text { is a limit ordinal }\right\} .
$$

Recall that an $\omega_{1}$-oracle is any sequence $\mathcal{M}=\left\langle M_{\delta}: \delta \in \Gamma\right\rangle$, where $M_{\delta}$ is a countable transitive model of $\mathrm{ZFC}^{-}$(that is, ZFC without the power set axiom) with the property that $\delta+1 \subset M_{\delta}, \delta$ is countable in $M_{\delta}$, and the set $\left\{\delta \in \Gamma: A \cap \delta \in M_{\delta}\right\}$ is stationary in $\omega_{1}$ for every $A \subset \omega_{1}$. With each $\omega_{1}$-oracle $\mathcal{M}=\left\langle M_{\delta}: \delta \in \Gamma\right\rangle$ there is associated a filter $D_{\mathcal{M}}$ generated by the sets $I_{\mathcal{M}}(A)=\left\{\delta \in \Gamma: A \cap \delta \in M_{\delta}\right\}$ for $A \subset \omega_{1}$. It is proved in [Sh, Claim 1.4] that $D_{\mathcal{M}}$ is a proper normal filter containing every closed unbounded subset of $\Gamma$. We will also need the following fact, which, for our purposes, can be viewed as the definition of the $\mathcal{M}$-cc property.

FACT 14 ([CS, Fact 4]). Let $P$ be a forcing notion of cardinality $\leq \omega_{1}$, $e: P \rightarrow \omega_{1}$ be one-to-one, and $\mathcal{M}=\left\langle M_{\delta}: \delta \in \Gamma\right\rangle$ be an $\omega_{1}$-oracle. If there exists a $C \in D_{\mathcal{M}}$ such that for every $\delta \in \Gamma \cap C$,

$e^{-1}(E)$ is predense in $P$ for every set $E \in M_{\delta} \cap \mathcal{P}(\delta)$ for which $e^{-1}(E)$ is predense in $e^{-1}(\{\gamma: \gamma<\delta\})$,

then $P$ has the $\mathcal{M}$-cc property.

Let $\mathcal{K}$ be the family of all sequences $\bar{h}=\left\langle h_{\xi}: \xi \in \Gamma\right\rangle$ such that each $h_{\xi}$ is a function from a countable set $D_{\xi} \subset \mathbb{R}$ onto $R_{\xi} \subset \mathbb{R}$ such that $h_{\xi}$ is dense in $\mathbb{R}^{2}$ and

$$
D_{\xi} \cap D_{\eta}=R_{\xi} \cap R_{\eta}=\emptyset \quad \text { for any distinct } \xi, \eta \in \Gamma \text {. }
$$


For each $\bar{h} \in \mathcal{K}$ we will define a forcing notion $Q_{\bar{h}}$. The forcing $Q_{\mathcal{B}}$ required in Lemma 2 will be chosen as $Q_{\bar{h}}$ for some $\bar{h} \in \mathcal{K}$. So let $\mathcal{H}$ be the family of all strictly increasing functions from finite subsets of $\mathbb{R}$ into $\mathbb{R}$ and fix an $\bar{h} \in \mathcal{K}$. Then $Q_{\bar{h}}$ is defined as

$$
Q_{\bar{h}}=\left\{h \in \mathcal{H}: h \subset \bigcup_{\xi \in \Gamma} h_{\xi} \&\left|h \cap h_{\xi}\right| \leq 1 \text { for every } \xi \in \Gamma\right\}
$$

and is ordered by reverse inclusion. In what follows we will use the following basic property of $Q_{\bar{h}}$.

FACT 15. Let $\bar{h}=\left\langle h_{\xi}: \xi \in \Gamma\right\rangle \in \mathcal{K}$ and $f=\bigcup H$, where $H$ is a $V$ generic filter over $Q_{\bar{h}}$. Then $f$ is a strictly increasing function from a dense subset $D$ of $\mathbb{R}$ onto a dense subset of $\mathbb{R}$. In particular, $f$ can be uniquely extended to an increasing homeomorphism $\widetilde{f}$ of $\mathbb{R}$.

Proof. Clearly $f$ is a strictly increasing function from a subset $D$ of $\mathbb{R}$ onto a subset $R$ of $\mathbb{R}$. Thus, it is enough to show that $D$ and $R$ are dense in $\mathbb{R}$. So let $U \neq \emptyset$ be open in $\mathbb{R}$ and notice that the set

$$
D=\left\{h \in Q_{\bar{h}}: \operatorname{dom}(h) \cap U \neq \emptyset\right\}
$$

is dense in $Q_{\bar{h}}$. Indeed, if $h_{0} \in Q_{\bar{h}}$ is such that $\operatorname{dom}\left(h_{0}\right) \cap U=\emptyset$ then we can find $\xi \in \Gamma$ such that $h \cap h_{\xi}=\emptyset$. Since the graph of $h_{\xi}$ is dense in $\mathbb{R}^{2}$ we can find $\langle x, y\rangle \in h_{\xi}$ such that $x \in U$ and $h=h_{0} \cup\{\langle x, y\rangle\}$ is strictly increasing. Then $h \in D$ extends $h_{0}$. Similarly we can prove that the set

$$
\left\{h \in Q_{\bar{h}}: \text { range }(h) \cap U \neq \emptyset\right\}
$$

is dense in $Q_{\bar{h}}$. The rest follows from the genericity of $H$.

Now let $\mathcal{B}=\left\{B_{\xi}: \xi<\omega_{1}\right\}$ be as in the lemma and fix an $\omega_{1}$-oracle $\mathcal{M}=\left\langle M_{\delta}: \delta \in \Gamma\right\rangle$. By Fact 15 in order to prove Lemma 2 it is enough to find an $\bar{h}=\left\langle h_{\xi}: \xi \in \Gamma\right\rangle \in \mathcal{K}$ such that

$$
Q_{\mathcal{B}}=Q_{\bar{h}} \text { is } \mathcal{M} \text {-cc }
$$

and $Q_{\bar{h}}$ forces that, in $V[H]$,

$$
\text { the set } \pi\left(f \cap B_{\xi}\right) \text { is nowhere meager for every } \xi<\omega_{1} \text {, }
$$

where the function $f$ is as in Fact 15 . To define $\bar{h}$ we will construct a sequence $\left\langle\left\langle x_{\alpha}, y_{\alpha}\right\rangle \in \mathbb{R}^{2}: \alpha<\omega_{1}\right\rangle$ aiming at $h_{\xi}=\left\{\left\langle x_{\xi+n}, y_{\xi+n}\right\rangle: n<\omega\right\}$, where $\xi \in \Gamma$. So let $\mathcal{U} \not \nexists \emptyset$ be a standard countable basis for $\mathbb{R}$ and for every $\xi \in \Gamma$ let $\left\langle\left\langle U_{n}^{\xi}, V_{n}^{\xi}, \zeta_{n}^{\xi}\right\rangle: n<\omega\right\rangle$ be a fixed enumeration of $\mathcal{U} \times \mathcal{U} \times \xi$. Points $\left\langle x_{\xi+n}, y_{\xi+n}\right\rangle$ are chosen inductively in such a way that

(i) $\left\langle x_{\xi+n}, y_{\xi+n}\right\rangle$ is a Cohen real over $M_{\delta}\left[\left\langle\left\langle x_{\alpha}, y_{\alpha}\right\rangle: \alpha<\xi+n\right\rangle\right]$ for every $\delta \leq \xi, \delta \in \Gamma$, that is, $\left\langle x_{\xi+n}, y_{\xi+n}\right\rangle$ is outside all meager subsets of $\mathbb{R}^{2}$ which are coded in $M_{\delta}\left[\left\langle\left\langle x_{\alpha}, y_{\alpha}\right\rangle: \alpha<\xi+n\right\rangle\right]$;

(ii) $\left\langle x_{\xi+n}, y_{\xi+n}\right\rangle \in\left(U_{n}^{\xi} \times V_{n}^{\xi}\right) \cap B_{\zeta_{n}^{\xi}}$. 
The choice of $\left\langle x_{\xi+n}, y_{\xi+n}\right\rangle$ is possible since the sets $\left(U_{n}^{\xi} \times V_{n}^{\xi}\right) \cap B_{\zeta_{n}^{\xi}}$ are non-meager and each time we need to avoid only countably many meager sets. Condition (ii) guarantees that the graph of each $h_{\xi}$ will be dense in $\mathbb{R}^{2}$. Note also that if $\Gamma \ni \delta \leq \alpha_{0}<\ldots<\alpha_{k-1}$, where $k<\omega$, then (by the product lemma in $M_{\delta}$ )

$$
\left\langle\left\langle x_{\alpha_{i}}, y_{\alpha_{i}}\right\rangle: i<k\right\rangle \text { is an } M_{\delta} \text {-generic Cohen real in }\left(\mathbb{R}^{2}\right)^{k} .
$$

For $h \in \mathcal{H}$ and $0<k<\omega$ let $U(h, k)$ stand for the set of all sequences $\left\langle\left\langle a_{i}, b_{i}\right\rangle \notin h: i<k\right\rangle \in\left(\mathbb{R}^{2}\right)^{k}$ such that $h \cup\left\{\left\langle a_{i}, b_{i}\right\rangle: i<k\right\} \in \mathcal{H}$. Clearly $U(h, k)$ is an open subset of $\left(\mathbb{R}^{2}\right)^{k}$. In fact, it can be easily proved that if $h=\left\{\left\langle x_{j}, y_{j}\right\rangle: 0<j<m\right\}$, where $x_{1}<\ldots<x_{m-1}$, then $\left\langle\left\langle a_{i}, b_{i}\right\rangle: i<k\right\rangle$ belongs to $U(h, k)$ if and only if $\left\{\left\langle a_{i}, b_{i}\right\rangle: i<k\right\} \in \mathcal{H}$ is disjoint from $h$ and

$$
\left\{\left\langle a_{i}, b_{i}\right\rangle: i<k\right\} \subset \bigcup_{j<m}\left(x_{j}, x_{j+1}\right) \times\left(y_{j}, y_{j+1}\right),
$$

where $x_{0}=y_{0}=-\infty$ and $x_{m}=y_{m}=\infty$. In particular, if $0<j_{0}<\ldots<$ $j_{k-1}<m$ and sets $W_{i} \ni\left\langle x_{j_{i}}, y_{j_{i}}\right\rangle, i<k$, are open in $\mathbb{R}^{2}$ then there are non-empty open sets $V_{i} \subset W_{i}$ such that

$$
\prod_{i<k} V_{i} \subset U(h, k) .
$$

For $\delta \in \Gamma$ let $\left(Q_{\bar{h}}\right)^{\delta}=\left\{h \in Q_{\bar{h}}: h \subset \bigcup_{\zeta<\delta} h_{\zeta}\right\}$.

FACT 16. Let $\delta \in \Gamma$ and let $E \in M_{\delta}$ be a predense subset of $\left(Q_{\bar{h}}\right)^{\delta}$. Then for every $k<\omega$ and $h \in\left(Q_{\bar{h}}\right)^{\delta}$ the open set

$$
B_{h}^{k}=\bigcup\left\{U(g, k): g \in\left(Q_{\bar{h}}\right)^{\delta} \text { extends } h \text { and some } h_{0} \in E\right\}
$$

is dense in $U(h, k)$.

Proof. Let $\left\langle\left\langle a_{i}, b_{i}\right\rangle: i<k\right\rangle \in U(h, k) \subset\left(\mathbb{R}^{2}\right)^{k}$ and let $W$ be an open subset of $U(h, k)$ containing $\left\langle\left\langle a_{i}, b_{i}\right\rangle: i<k\right\rangle$. We need to show that $W$ intersects $U(g, k)$ for some $g \in Q_{\bar{h}}$ extending $h$ and an $h_{0} \in E$. Decreasing $W$ if necessary, we can assume that it is of the form $\prod_{i<k} W_{i}$. Since $h_{1}=h \cup\left\{\left\langle a_{i}, b_{i}\right\rangle: i<k\right\} \in \mathcal{H}$, by (13) there are open sets $V_{i} \subset W_{i}$ for which $\prod_{i<k} V_{i} \subset U(h, k) \cap W$. In particular, for any choice of points $\left\langle c_{i}, d_{i}\right\rangle \in V_{i}$ we have $h_{1} \cup\left\{\left\langle c_{i}, d_{i}\right\rangle: i<k\right\} \in \mathcal{H}$. Since all functions $h_{\xi}$ are dense in $\mathbb{R}^{2}$, we can choose points $\left\langle c_{i}, d_{i}\right\rangle$ from distinct functions $h_{\xi}$ in such a way that $h_{2}=h \cup\left\{\left\langle c_{i}, d_{i}\right\rangle: i<k\right\} \in\left(Q_{\bar{h}}\right)^{\delta}$. Since $E$ is predense in $\left(Q_{\bar{h}}\right)^{\delta}$, there exists a $g \in\left(Q_{\bar{h}}\right)^{\delta}$ extending $h_{2} \leq h$ and some $h_{0} \in E$. But $\left\{\left\langle c_{i}, d_{i}\right\rangle: i<k\right\} \subset g$, so by (13), there are non-empty open sets $U_{i} \subset V_{i}$ for which $\prod_{i<k} U_{i} \subset U(g, k) \cap W$. 
Now we are ready to prove (10), that is, that $Q_{\bar{h}}$ is $\mathcal{M}$-cc. So fix a bijection $e: Q_{\bar{h}} \rightarrow \omega_{1}$ and let

$$
C=\left\{\delta \in \Gamma:\left(Q_{\bar{h}}\right)^{\delta}=e^{-1}(\delta) \in M_{\delta}\right\} .
$$

Then $C \in D_{\mathcal{M}}$. (See e.g. [Sh, Claim 1.4(4)].) Take a $\delta \in C$ and fix an $E \subset \delta$, $E \in M_{\delta}$, for which $e^{-1}(E)$ is predense in $\left(Q_{\bar{h}}\right)^{\delta}$. By Fact 14 it is enough to show that

$$
e^{-1}(E) \text { is predense in } Q_{\bar{h}} \text {. }
$$

So take $h_{0}$ from $Q_{\bar{h}}$, put $h=h_{0}\left\lceil\bigcup_{\eta<\delta} D_{\eta}\right.$ and $h_{1}=h_{0} \backslash h$, and notice that the condition $h$ belongs to $\left(Q_{\bar{h}}\right)^{\delta}$. Assume that $h_{1}=\left\{\left\langle x_{i}, y_{i}\right\rangle: i<k\right\}$, where $x_{0}<\ldots<x_{k-1}$. So $\left\langle\left\langle x_{i}, y_{i}\right\rangle: i<k\right\rangle \in U(h, k)$. By Fact 16 the set $U(h, k) \backslash B_{h}^{k}$ is nowhere dense and belongs to $M_{\delta}$ (as it is defined from $\left.\left(Q_{\bar{h}}\right)^{\delta} \in M_{\delta}\right)$. Hence, by $(12),\left\langle\left\langle x_{i}, y_{i}\right\rangle: i<k\right\rangle$ cannot belong to this set, so $\left\langle\left\langle x_{i}, y_{i}\right\rangle: i<k\right\rangle \in B_{h}^{k}$. In particular, there is a $g \in\left(Q_{\bar{h}}\right)^{\delta}$ extending $h$ and some $h_{0} \in e^{-1}(E)$ such that $\left\langle\left\langle x_{i}, y_{i}\right\rangle: i<k\right\rangle \in U(g, k)$. But then $g \cup h_{1}$ belongs to $Q_{\bar{h}}$ and extends $h$ and $h_{0}$. This finishes the proof of (10). The proof of (11) is similar.

So fix a $\zeta<\omega_{1}$. We will prove that $\pi\left(f \cap B_{\zeta}\right)$ is nowhere meager in $\mathbb{R}$. Suppose not. Then there exists a $U^{*} \in \mathcal{U}$ such that $\pi\left(f \cap B_{\zeta}\right)$ is meager in $U^{*}$. Let a condition $h^{*} \in Q_{\bar{h}}$ and $Q_{\bar{h}^{-} \text {-names }} \dot{U}_{m}$, for $m<\omega$, be such that

$h^{*} \Vdash_{Q_{\bar{h}}}$ "each $\dot{U}_{m}$ is an open dense subset of $U^{*}$ and

$$
\pi\left(f \cap B_{\zeta}\right) \cap \bigcap_{m<\omega} \dot{U}_{m}=\emptyset . "
$$

For each $m<\omega$, since $h^{*}$ forces that $\dot{U}_{m}$ is an open dense subset of $U^{*}$, for every subset $U \in \mathcal{U}$ of $U^{*}$ there is a subset $V \in \mathcal{U}$ of $U$ and a maximal antichain $\left\langle h_{V, k}^{m}: k<\kappa_{V}^{m}\right\rangle$ in $Q_{\bar{h}}$ such that each $h_{V, k}^{m}$ forces that $V \subset \dot{U}_{m}$. Note that each of these antichains must be countable, since the forcing notion $Q_{\bar{h}}$ is $\mathcal{M}$-cc and therefore ccc. Combining all these antichains we find a $\mathcal{V} \subset \mathcal{U}$ and a sequence $\left\langle h_{V, k}^{m} \in Q_{\bar{h}}: m<\omega, V \in \mathcal{V}, k<\kappa_{V}^{m}\right\rangle$ such that

- $\kappa_{V}^{m} \leq \omega$,

- $h_{V, k}^{m} \Vdash_{Q_{\bar{h}}} " V \subseteq \dot{U}_{m}$ ",

- for every $m<\omega, h \in Q_{\bar{h}}$ extending $h^{*}$, and every subset $U \in \mathcal{U}$ of $U^{*}$ there is a subset $V \in \mathcal{V}$ of $U$ and a $k<\kappa_{V}^{m}$ such that the conditions $h$ and $h_{V, k}^{m}$ are compatible.

Note that for sufficiently large $\delta \in \Gamma$ we have $h_{V, k}^{m} \in\left(Q_{\bar{h}}\right)^{\delta}$ for all $m<\omega$, $V \in \mathcal{V}$, and $k<\kappa_{V}^{m}$. Now, by the definition of $\omega_{1}$-oracle, the set $B_{0}$ of all $\delta \in \Gamma$ for which

$$
\left\langle h_{V, k}^{m} \in Q_{\bar{h}}: m<\omega, V \in \mathcal{V}, k<\kappa_{V}^{m}\right\rangle \in M_{\delta} \quad \text { and } \quad\left(Q_{\bar{h}}\right)^{\delta} \in M_{\delta}
$$

is stationary in $\omega_{1}$. Thus, choose a $\delta>\zeta$ from $B_{0}$ and let $U, W \in \mathcal{U}$ be such that $U \subset U^{*}$ and $h^{*} \cup\{\langle x, y\rangle\} \in \mathcal{H}$ for every $\langle x, y\rangle \in U \times W$. Using clause 
(ii) of the choice of $x_{\alpha}$ 's we may find an $n<\omega$ such that $\left\langle x_{\delta+n}, y_{\delta+n}\right\rangle \in$ $(U \times W) \cap B_{\zeta}$. Then $h_{0}=h^{*} \cup\left\{\left\langle x_{\delta+n}, y_{\delta+n}\right\rangle\right\} \in Q_{\bar{h}}$ extends $h^{*}$ and $h_{0} \Vdash$ " $x_{\delta+n} \in U^{*} \cap \pi\left(f \cap B_{\zeta}\right)$ " since $\left\langle x_{\delta+n}, y_{\delta+n}\right\rangle \in f$. We will show that

$$
h_{0} \Vdash " x_{\delta+n} \in \bigcap_{m<\omega} \dot{U}_{m} ",
$$

which contradicts the choice of $h^{*}$. Assume that this is not the case. Then there exist $i<\omega$ and $h_{1} \in Q_{\bar{h}}$ stronger than $h_{0}$ such that $h_{1} \Vdash " x_{\delta+n} \notin \dot{U}_{i}$ ". Define $h=h_{1} \uparrow\left\{x_{\alpha}: \alpha<\delta\right\} \in\left(Q_{\bar{h}}\right)^{\delta}$ and $h_{1} \backslash h=\left\{\left\langle a_{l}, b_{l}\right\rangle: l<m\right\}$, where $a_{0}<\ldots<a_{m-1}$. Let $j<m$ be such that $\left\langle x_{\delta+n}, y_{\delta+n}\right\rangle=\left\langle a_{j}, b_{j}\right\rangle$. Consider the set $Z$ of all $\left\langle\left\langle z_{l}, z_{l}^{\prime}\right\rangle: l<m\right\rangle \in\left(\mathbb{R}^{2}\right)^{m}$ for which

- there exist $V \in \mathcal{V}, k<\kappa_{V}^{i}$, and $g \in\left(Q_{\bar{h}}\right)^{\delta}$ such that $z_{j} \in V, g$ extends $h$ and $h_{V, k}^{i}$, and $\left\langle\left\langle z_{l}, z_{l}^{\prime}\right\rangle: l<m\right\rangle \in U(g, m)$.

Claim. The set $Z$ belongs to the model $M_{\delta}$ and it is an open dense subset of $K=\left\{\left\langle\left\langle z_{l}, z_{l}^{\prime}\right\rangle: l<m\right\rangle \in U(h, m): z_{j} \in U^{*}\right\}$.

Proof. It should be clear that $Z$ is (coded) in $M_{\delta}$. (Remember the choice of $\delta$.) It is also obvious that $Z$ is open. To show that it is dense in $U(h, m)$ we proceed as in the proof of Fact 16 . Let $\left\langle\left\langle c_{l}, d_{l}\right\rangle: l<m\right\rangle \in K$ and $W$ be an open subset of $K$ containing $\left\langle\left\langle c_{l}, d_{l}\right\rangle: l<m\right\rangle$. We need to show that $W \cap Z \neq \emptyset$. As in the proof of Fact 16 we can find an open set $\prod_{l<m}\left(V_{l} \times U_{l}\right) \subset K \cap W$ and points $\left\langle c_{l}, d_{l}\right\rangle \in V_{l} \times U_{l}$ such that $h_{2}=h \cup\left\{\left\langle c_{l}, d_{l}\right\rangle: l<m\right\} \in\left(Q_{\bar{h}}\right)^{\delta}$. Since $h_{2} \in\left(Q_{\bar{h}}\right)^{\delta}$ extends $h^{*}$ and $V_{j}$ is an open subset of $U^{*}$, there is a subset $V \in \mathcal{V}$ of $V_{j}$ and a $k<\kappa_{V}^{i}$ such that the conditions $h_{2}$ and $h_{V, k}^{i}$ are compatible. Let $g \in Q_{\bar{h}}$ extend $h_{2}$ and $h_{V, k}^{i}$. Since $h_{2}, h_{V, k}^{i} \in\left(Q_{\bar{h}}\right)^{\delta}$ we can assume that $g \in\left(Q_{\bar{h}}\right)^{\delta}$. But $\left\{\left\langle c_{l}, d_{l}\right\rangle: l \stackrel{<}{<}\right\} \subset g$, so by (13), there are non-empty open sets $U_{l}^{\prime} \subset U_{l}$ and $V_{l}^{\prime} \subset V_{l}$ for which $\prod_{l<m}\left(U_{i}^{\prime} \times V_{i}^{\prime}\right) \subset U(g, m) \cap \prod_{l<m}\left(V_{l} \times U_{l}\right)$. Thus, $\emptyset \neq \prod_{l<m}\left(U_{i}^{\prime} \times V_{i}^{\prime}\right) \subset Z \cap W$. This completes the proof of the Claim.

Now, $\left\langle\left\langle a_{l}, b_{l}\right\rangle: l<m\right\rangle$ belongs to $K$. Since by the Claim, $K \backslash Z \in M_{\delta}$ is nowhere dense, by (12) we conclude that this point does not belong to $K \backslash Z$. So $\left\langle\left\langle a_{l}, b_{l}\right\rangle: l<m\right\rangle \in Z$. But this means that there exist $g \in\left(Q_{\bar{h}}\right)^{\delta}$ and $V \in \mathcal{V}$ such that:

- $g \leq h, g \Vdash$ " $V \subseteq \dot{U}_{i} "$,

- $\left\langle\left\langle a_{l}, b_{l}\right\rangle: l<m\right\rangle \in U(h, m)$, and $x_{\delta+n}=a_{j} \in V$.

But then $h_{3}=g \cup\left\{\left\langle a_{l}, b_{l}\right\rangle: l<m\right\}$ belongs to $Q_{\bar{h}}$ and extends both $g$ and $h_{1}$. So $h_{3}$ forces that $x_{\delta+n}=a_{j} \in V \subseteq \dot{U}_{i}$, contradicting our assumption that $h_{1} \Vdash$ " $x_{\delta+n} \notin \dot{U}_{i}$ ". This finishes the proof of (11) and of Lemma 2 . 
5. A conjecture. We believe that Corollary 5 cannot be proved in ZFC. We believe the following is consistent with ZFC.

(C) For every nowhere meager set $A \subset[0,1]$ there exists a continuous Peano-like function $p$ from $[0,1]$ onto $[0,1]^{2}$ such that for every $x \in$ $[0,1]$ the set $p[A] \cap(\{x\} \times[0,1])$ is non-meager in $\{x\} \times[0,1]$.

Proposition 17. If $(\mathrm{C})$ holds then for every nowhere meager set $A \subset \mathbb{R}$ there is an $f \in \mathcal{C}_{0}$ such that $\pi[(f+c) \cap(A \times A)] \notin \mathcal{M}$ for every $c \in \mathbb{R}$.

Proof. Let $A \subset \mathbb{R}$ be nowhere meager, $f$ be as in condition (C), and let $f_{0}=\pi \circ p:[0,1] \rightarrow[0,1]$. Since there exists a meager set $M \subset[0,1]$ such that $p\left\lceil[0,1] \backslash M\right.$ is a homeomorphism between $[0,1] \backslash M$ and $[0,1]^{2} \backslash p[M]$, for every $c \in[0,1]$ we have

$$
\pi\left[\left(f_{0}+c\right) \cap(A \times A)\right]=\pi\left[f_{0} \cap(A \times(A-c))\right]=A \cap\left(f_{0}\right)^{-1}(A-c) \notin \mathcal{M}
$$

since $A \cap\left(f_{0}\right)^{-1}(A-c)=A \cap p^{-1}\left(\pi^{-1}(A-c)\right)=A \cap p^{-1}((A-c) \times[0,1])$ and this last set is equal, modulo $\mathcal{M}$, to $p^{-1}(p[A] \cap((A-c) \times[0,1]))$ while, by $(\mathrm{C})$, the set $p[A] \cap((A-c) \times[0,1])$ is not meager (by the Kuratowski-Ulam theorem). To get a function from $\mathbb{R}$ to $\mathbb{R}$ glue countably many shifts of $f_{0}$.

\section{References}

[BH] T. Bartoszyński and L. Halbeisen, There are big symmetric planar sets meeting polynomials at just finitely many points, preprint.

[CG] J. Ceder and D. K. Ganguly, On projections of big planar sets, Real Anal. Exchange 9 (1983-84), 206-214.

[Ci] K. Ciesielski, Set Theory for the Working Mathematician, London Math. Soc. Stud. Texts 39, Cambridge Univ. Press, 1997.

[CMP] K. Ciesielski, A. Millán and J. Pawlikowski, Uncountable $\gamma$-sets under axiom CPA $_{\text {cube }}^{\text {game }}$, Fund. Math. 176 (2003), 143-155. (Preprint ${ }^{\star}$ available.) $\left(^{1}\right)$

[CP1] K. Ciesielski and J. Pawlikowski, Covering Property Axiom $\mathrm{CPA}_{\text {cube }}$ and its consequences, ibid., 63-75. (Preprint ${ }^{\star}$ available.)

[CP2] - - - Crowded and selective ultrafilters under the Covering Property Axiom, J. Appl. Anal. 9 (2003), 19-55. (Preprint* available.)

[CP3] - - - Covering Property Axiom CPA, version of January 2003, work in progress*

[CS] K. Ciesielski and S. Shelah, Category analog of sup-measurability problem, J. Appl. Anal. 6 (2000), 159-172. (Preprint* available.)

[Da] R. O. Davies, Second category $E$ with each $\operatorname{proj}\left(\mathbb{R}^{2} \backslash E^{2}\right)$ dense, Real Anal. Exchange 10 (1984-85), 231-232.

[Ku] M. Kuczma, An Introduction to the Theory of Functional Equations and Inequalities, PWN, Warszawa, 1985.

[N1] T. Natkaniec, On category projections of cartesian product $A \times A$, Real Anal. Exchange 10 (1984-85), 233-234.

[N2] - On projections of planar sets, ibid. 11 (1985-86), 411-416.

$\left({ }^{1}\right)$ Papers marked by * are available in electronic form from Set Theoretic Analysis Web Page: http://www.math.wvu.edu/ ${ }^{\sim}$ kcies/STA/STA.html. 
[Sh] S. Shelah, Proper and Improper Forcing, Perspectives in Math. Logic, Springer, 1998.

Department of Mathematics

West Virginia University

Morgantown, WV 26506-6310, U.S.A.

E-mail: K_Cies@math.wvu.edu

Web: http://www.math.wvu.edu/ ${ }^{\sim}$ kcies
Department of Mathematics

Gdańsk University

Wita Stwosza 57

80-952 Gdańsk, Poland

E-mail: mattn@math.univ.gda.pl

Received 7 October 2002;

in revised form 20 August 2003 